\title{
Hookworm in the Northern Territory: down but not out
}

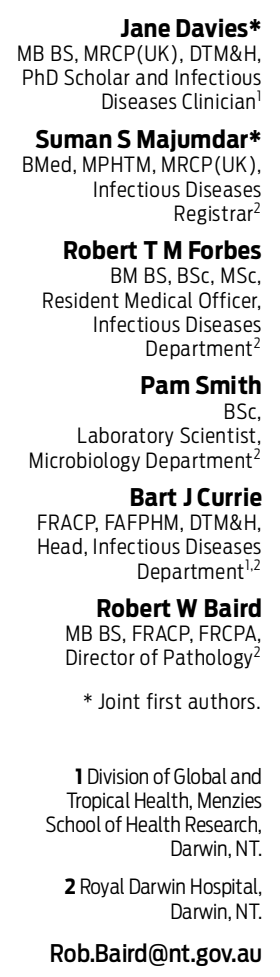

MJA 2013; 198: 278-281 doi:10.5694/mjal2.11615 ookworms (Ancylostoma duodenale and Necator americanus) are thought to infect 740 million people worldwide. ${ }^{1}$ Most infections occur in low- and middleincome countries where the root causes are socioeconomic vulnerability and limited access to safe water, good hygiene and adequate sanitation. Hookworm, along with other soil-transmitted helminthiases, is included in the World Health Organization category of neglected tropical diseases. A. duodenale is thought to be the exclusive species in the Northern Territory and is associated with the greatest intestinal blood loss. . $^{2,3}$

The major consequence of hookworm infection (HWI) is iron-deficiency anaemia, which is responsible for significant morbidity, particularly in children and pregnant women. In children, HWI is associated with impaired nutritional status and physical and mental development. ${ }^{4} \mathrm{HWI}$ is considered a public health problem in a country when the prevalence of infection in children aged 1-14 years is $>1 \%$. The WHO's 2010 global target of $75 \%$ coverage of preventive chemotherapy or "deworming" in such countries was not met and has been extended to $2020 .^{1}$ A recent Cochrane review concluded that the evidence base supporting the effectiveness of routine deworming programs was small and insufficient using Cochrane principles, ${ }^{5}$ but there is considerable historical support for the role of mass drug therapy for HWI, in parallel with sanitation and hygiene initiatives. ${ }^{1}$

Although virtually eradicated from the non-Indigenous Australian population by the 1960s, HWI has persisted in Indigenous Australians in remote communities and in immigrants from endemic countries. ${ }^{2}$ After successful lobbying by NT clinicians for registration of albendazole in Australia, ${ }^{6}$ instead of the inferior pyrantel, the Indigenous community children's deworming program (CCDP) with albendazole commenced in 1995 in the NT. ${ }^{7}$ The Central Australian Rural Practitioners Association (CARPA)

\section{Abstract}

Objectives: To determine the prevalence and trends of human hookworm infection ( $\mathrm{HWI}$ ) in the Northern Territory over the past 10 years, and to assess the influence of the community children's deworming program (CCDP).

Design, patients and setting: A retrospective observational analysis of consecutive microbiologically confirmed cases of $\mathrm{HWI}$ in patients diagnosed at NT government health care facilities and the main private laboratory servicing the NT between January 2002 and July 2012.

Main outcome measures: Annual prevalence of HWI (2002-2011); age, sex, Indigenous status, residence, haemoglobin level and eosinophil count of patients with $\mathrm{HWl}$; and proportion of patients within the CCDP target population (children aged 6 months to 16 years, who should receive 6-monthly albendazole).

Results: From 64691 faecal samples examined during the study period, hookworm was detected in 112 patients. There was a downward trend in the annual prevalence of HWI, falling from 14.0 cases per 100000 population (95\% $\mathrm{Cl}, 8.8-19.2)$ in 2002 to 2.2 per 100000 population $(95 \% \mathrm{Cl}, 0.3-4.1)$ in 2011. Only 16 patients (14.3\%) fell within the CCDP target population. Seventy-one patients (63.4\%) were living in remote communities, and 94 (84.7\%) were recorded as Indigenous Australians.

Conclusions: The prevalence of HWI in the NT reduced over the 10-year period. HWI predominantly occurs in individuals outside the CCDP target population. Our data support continuation of the CCDP in conjunction with improvements in housing, health hardware and health promotion. Continued use of albendazole in individuals beyond the CCDP may facilitate the eventual eradication of $\mathrm{HWI}$ from the NT.

Standard treatment manual recommends that in areas where HWI is common, children aged 6 months to 16 years should have a single dose of albendazole twice a year (or pyrantel if pregnant). ${ }^{8}$ It suggests administration either side of the wet season, in conjunction with routine child health assessments or school-age screening.

Recent epidemiological data on the prevalence of HWI in the NT are not available, ${ }^{9}$ and there are no published data on the implementation and effectiveness of the CCDP. The objectives of this study were to determine the prevalence and trends of human HWI in the NT over the past 10 years and to assess the influence of the CCDP.

\section{Methods}

We conducted a retrospective observational analysis of consecutive microbiologically confirmed cases of HWI in the NT between January 2002 and July 2012. Ethics approval for the study was obtained from the Human Research Ethics Committee of the NT
Department of Health and Menzies School of Health Research (HREC2012-1842).

\section{Data collection}

Cases were identified from the NT government pathology laboratories information system, LabTrak, which covers all NT government health care facilities, including five hospitals, two correctional centres and more than 50 remote clinics, that collectively serve a current population of about $235000 .^{10}$ We also identified cases from data obtained from Western Diagnostic Pathology, the major private provider of pathology services to remote clinics and Aboriginal community-controlled health services in the NT. Data were not available from the two other private providers of pathology services to the NT. Each pathology laboratory examined faecal specimens for hookworm eggs by wet mount microscopy and a concentration method. ${ }^{11}$

Data on identified HWI cases were linked to NT government electronic health records by medical record number to collect information on 
1 Demographic and laboratory parameters of patients with hookworm infection,

Northern Territory, January 2002 - July 2012

\begin{tabular}{|c|c|c|c|c|}
\hline \multirow[b]{2}{*}{ Parameter } & \multirow[b]{2}{*}{ All patients } & \multicolumn{3}{|c|}{ Age group } \\
\hline & & $<17$ years & $\geqslant 17$ years & $P$ \\
\hline Number & 112 & $17(15.2 \%)$ & $95(84.8 \%)$ & \\
\hline Sex & & & & 0.60 \\
\hline Female & 51 (45.5\%) & $9(52.9 \%)$ & $42(44.2 \%)$ & \\
\hline Male & $61(54.5 \%)$ & $8(47.1 \%)$ & $53(55.8 \%)$ & \\
\hline Indigenous status* & & & & 0.46 \\
\hline Indigenous & 94 (84.7\%) & 16 (94.1\%) & 78 (83.0\%) & \\
\hline Non-Indigenous & $17(15.3 \%)$ & $1(5.9 \%)$ & $16(17.0 \%)$ & \\
\hline Patient residence $^{\dagger}$ & & & & 0.03 \\
\hline Urban & $23(20.5 \%)$ & 0 & $23(24.2 \%)$ & \\
\hline Remote & $71(63.4 \%)$ & $16(94.1 \%)$ & $55(57.9 \%)$ & \\
\hline Overseas & $13(11.6 \%)$ & 1 (5.9\%) & $12(12.6 \%)$ & \\
\hline Unknown & $5(4.5 \%)$ & 0 & $5(5.3 \%)$ & \\
\hline Anaemia $^{\ddagger}$ & $60(66.7 \%)$ & $12(75.0 \%)$ & $48(64.9 \%)$ & \\
\hline Median haemoglobin, g/L (IQR) ${ }^{\ddagger}$ & $94(75-120)$ & $91(56-108)$ & $94(76-122)$ & 0.18 \\
\hline Eosinophilia & $65(73.0 \%)$ & $12(75.0 \%)$ & $53(72.6 \%)$ & \\
\hline Median eosinophil count, $\times 10^{9} / \mathrm{L}(\mathrm{IQR})^{\S}$ & $1.1(0.3-1.8)$ & $1.9(0.8-3.4)$ & $0.9(0.3-1.6)$ & 0.02 \\
\hline
\end{tabular}

IQR = interquartile range. * Defined as Aboriginal or Torres Strait Islander. Indigenous status was not available for one patient aged $\geqslant 17$ years. $†$ Defined by Australian Standard Geographical

Classification. ${ }^{12} \ddagger$ Anaemia defined as haemoglobin level $<110 \mathrm{~g} / \mathrm{L}$. Data available for 90 patients

(16 patients $<17$ years and 74 patients $\geqslant 17$ years). $\$$ Eosinophilia defined as an eosinophil count

$\geqslant 0.5 \times 10^{9} / \mathrm{L}$. Data available for 89 patients ( 16 patients $<17$ years and 73 patients $\geqslant 17$ years).

patients' age, sex, Indigenous status, residence, haemoglobin level and eosinophil count at the time of diagnosis. Residence was determined from residential address, and remote (including very remote) areas of residence were defined as per the Australian Standard Geographical Classification. ${ }^{12}$ Anaemia was defined as a haemoglobin level $<110 \mathrm{~g} / \mathrm{L}$, and eosinophilia as an eosinophil count $\geqslant 0.5 \times 10^{9} / \mathrm{L}^{8}{ }^{8}$ We were unable to obtain data on anthelmintic treatment for individual patients. Attempts to obtain robust data regarding numbers of doses of albendazole dispensed per community clinic for the CCDP were not successful.

\section{Statistical analysis}

Data were entered into a Microsoft Access 2007 database and analysed using Stata, version 10 (StataCorp). Descriptive statistics are presented for all patients with HWI between January 2002 and July 2012. Differences between adults and children were determined using the Fisher exact test for categorical variables (sex, Indigenous status, residence) and MannWhitney tests for continuous non-normally distributed variables (haemoglobin level and eosinophil count). Level of significance was set at $P<0.05$.
The estimated annual prevalences for 2002 to 2011 are expressed as cases per 100000 population, with 95\% confidence intervals. The estimated prevalence for 2012 could not be calculated as the dataset only included cases up to the end of July. The estimated annual resident population for the NT was obtained from the Australian Bureau of Statistics. ${ }^{10}$

\section{Results}

From a total of 64691 faecal specimens examined during the period January 2002 - July 2012, hookworm eggs were identified in 134 samples from 112 patients. Four patients had a second positive faecal sample, all occurring within 8 months of the first detection.

The annual number of faecal samples tested across all NT laboratories was relatively constant, with a median of 5807 from 2002 to 2011 (range, 5379-9785). Only six patients (5.4\%) were diagnosed at Western Diagnostic Pathology, with the remainder diagnosed at government laboratories: $87(77.7 \%)$ at Royal Darwin Hospital, $14(12.5 \%)$ at Alice Springs Hospital, and five (4.5\%) at district laboratories. All patients except the six diagnosed at Western Diagnostic
Pathology were in hospital at the time of diagnosis. The details of the Western Diagnostic Pathology cases were incomplete, but three diagnoses were in Indigenous people living in remote communities.

The demographic characteristics, haemoglobin level and eosinophil count of the patients are shown in Box 1, stratified by age group. There were no statistical differences in Indigenous status or sex between children and adults in the sample, but there was a difference in residence. Median eosinophil count was significantly higher in children than in adults, but there was no significant difference in haemoglobin levels.

Most patients $(94 ; 84.7 \%)$ were Indigenous. Seventy-one patients $(63.4 \%)$ resided in remote areas, of whom 69 were Indigenous. Of the 17 children, 16 were Indigenous and one was from Sudan. All of the Indigenous children resided in remote communities. Twelve of the 13 patients from overseas were from Indonesia. Eleven patients $(9.8 \%)$ were of no fixed abode in Darwin (all Indigenous), and 23 (20.5\%) were diagnosed in prison facilities.

Ninety and 89 of the 112 patients had data available for haemoglobin level and eosinophil count, respectively. Sixty $(66.7 \%)$ were anaemic and $65(73.0 \%)$ had eosinophilia. Of note, no children and only four adults had neither of these markers present.

The annual prevalence of HWI by age, and the proportion of total faecal samples positive for hookworm are shown in Box 2, with the numbers of cases and population data in Box 3 . The estimated prevalence fell relatively steadily over time, from 14.0 per 100000 population (95\% CI, 8.8-19.2) in 2002 to 2.2 per 100000 population (95\% CI, 0.3-4.1) in 2011.

Only 16 patients $(14.3 \%)$ were in the target age range of the CCDP (6 months to 16 years). The one remaining child was under 6 months of age (5 months old and diagnosed in 2002). There have been only two cases of HWI in the $<17$-years age group since 2007 and none since 2010 (Box 3).

\section{Discussion}

We found a clear and consistent reduction in the number of microbio- 
2 Annual prevalence of microbiologically confirmed hookworm infection, by age group, and proportion of hookworm-positive faecal samples, Northern Territory, 2002-2012*

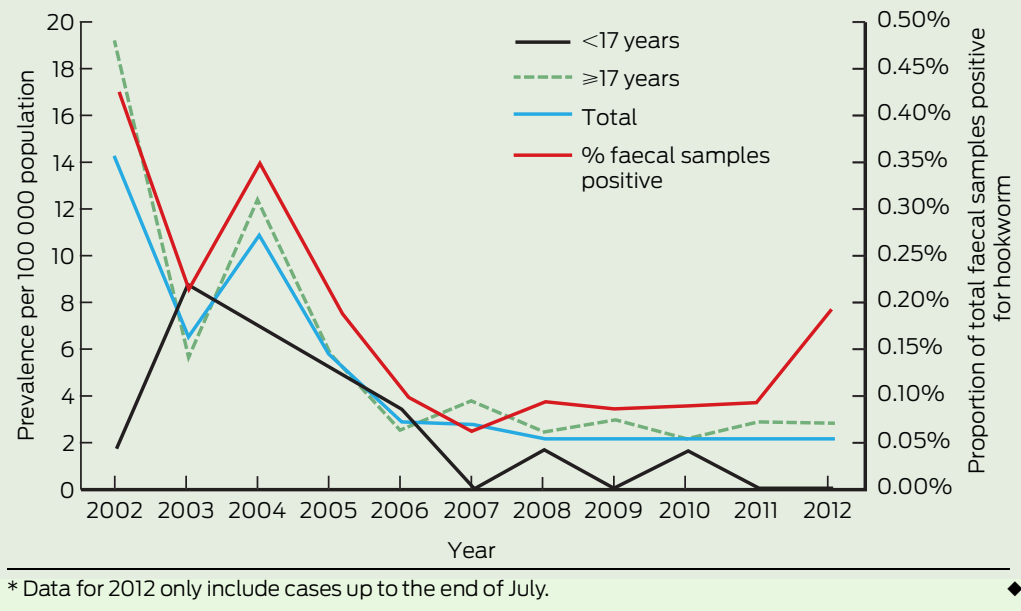

logically confirmed cases of HWI occurring in the NT from January 2002 to July 2012. Microbiologically confirmed HWI is now a rare event in the NT and occurs mainly in individuals outside the CCDP target group, particularly in adults, prisoners and homeless urban Indigenous people.

The epidemiology of HWI in Australia has been poorly documented since a national hookworm campaign and survey in 1919-1924 demonstrated a prevalence of $10.7 \%$ (14 067/ 130833 ) in endemic areas. ${ }^{2}$ The campaign, which involved treatment, health education and improving sanitation, was focused on non-Indigenous Australians and led to eradication from this population. The prevalence of HWI in the NT was 17\% in the 1924 survey (from 886 faecal samples), ${ }^{2}$ and $25 \%$ in a 1994 survey (from 300 samples) in a remote Indigenous community in east Arnhem Land (Aland K, Prociv P, Currie B, Jones $\mathrm{H}$. Intestinal parasite infections and anaemia in Arnhem Land Aboriginal community [unpublished abstract]. Australian Tropical Health and Nutrition Conference; Brisbane, Qld; 17-19 July 1997). We found the prevalence of HWI in the NT in 2011 had dropped to $2.2 \%$. This reduction has occurred since the commencement of the albendazole-based CCDP in 1995, and extremely low rates of HWI are now seen in its target population. However, the magnitude of the effect of the CCDP remains uncertain, as its implementation and coverage at community or regional level has never been monitored or evaluated.
In contrast to other soil-transmitted helminthiases in which the highest intensity and prevalence of infections occur in school-aged children, high-intensity HWIs predominantly occur in adults. ${ }^{3,13}$ In 1992, a survey in an isolated community in northern Western Australia found HWI prevalences of $93 \%$ in children aged 5-14 years and 77\% overall. ${ }^{14}$ After implementation of a hookworm control program, in which regular albendazole was given to children every 6 months and to adults annually, along with health education and environmental management measures, the overall HWI prevalence rate fell from $80 \%$ in 1993 to $2.6 \%$ in $1999 .{ }^{15}$ In our NT data, there was a marked reduction in the prevalence of adult HWI from 2002, despite adults not being targeted in the CCDP. This could in part be attributable to a carryover effect from early intense albendazole use in the CCDP in the 1990s and/or the common use of albendazole in the NT for individuals with anaemia or eosinophilia. ${ }^{8}$ The CARPA manual recommends empirical treatment with 3 days of albendazole (for hookworm, Trichuris trichiura and Strongyloides stercoralis infection) for anaemia, failure to thrive and significant diarrhoea in children. ${ }^{8}$

Our retrospective study has several limitations. The true prevalence rates are likely to have been underestimated, as systematic sampling was not undertaken. All except six patients were diagnosed in hospital, reflecting a selection bias towards individuals who seek health care, are acutely ill or

3 Annual hookworm infection cases and prevalence,* by age group, Northern Territory, 2002-2012

\begin{tabular}{|c|c|c|c|c|c|c|c|c|c|c|c|}
\hline & 2002 & 2003 & 2004 & 2005 & 2006 & 2007 & 2008 & 2009 & 2010 & 2011 & $2012^{\dagger}$ \\
\hline \multicolumn{12}{|l|}{ Age $<17$ years } \\
\hline Cases $(n=17)$ & 1 & 5 & 4 & 3 & 2 & 0 & 1 & 0 & 1 & 0 & 0 \\
\hline Population ${ }^{\ddagger}$ & 56990 & 56993 & 57123 & 57669 & 58159 & 58280 & 58721 & 59263 & 59316 & 58935 & 59232 \\
\hline Prevalence* & 1.8 & 8.8 & 7.0 & 5.2 & 3.4 & 0.0 & 1.7 & 0.0 & 1.7 & 0.0 & 0.0 \\
\hline \multicolumn{12}{|l|}{ Age $\geqslant 17$ years } \\
\hline Cases $(n=95)$ & 27 & 8 & 18 & 9 & 4 & 6 & 4 & 5 & 4 & 5 & 5 \\
\hline Population ${ }^{\ddagger}$ & 142421 & 143113 & 144940 & 148704 & 152468 & 156741 & 162214 & 167578 & 170999 & 172396 & 175604 \\
\hline Prevalence* & 19.0 & 5.6 & 12.4 & 6.1 & 2.6 & 3.8 & 2.5 & 3.0 & 2.3 & 2.9 & 2.8 \\
\hline \multicolumn{12}{|l|}{ All patients } \\
\hline Cases $(n=112)$ & 28 & 13 & 22 & 12 & 6 & 6 & 5 & 5 & 5 & 5 & 5 \\
\hline Population ${ }^{\ddagger}$ & 199411 & 200106 & 204919 & 206373 & 210627 & 215021 & 220935 & 226841 & 230315 & 231331 & 234836 \\
\hline $\begin{array}{l}\text { Prevalence* } \\
(95 \% \mathrm{Cl})\end{array}$ & $\begin{array}{c}14.0 \\
(8.8-19.2)\end{array}$ & $\begin{array}{c}6.5 \\
(3.0-10.0)\end{array}$ & $\begin{array}{c}10.9 \\
(6.3-15.4)\end{array}$ & $\begin{array}{c}5.8 \\
(2.5-9.1)\end{array}$ & $\begin{array}{c}2.8 \\
(0.6-5.1)\end{array}$ & $\begin{array}{c}2.8 \\
(0.6-5.0)\end{array}$ & $\begin{array}{c}2.3 \\
(0.3-4.3)\end{array}$ & $\begin{array}{c}2.2 \\
(0.3-4.1)\end{array}$ & $\begin{array}{c}2.2 \\
(0.3-4.1)\end{array}$ & $\begin{array}{c}2.2 \\
(0.3-4.1)\end{array}$ & $\begin{array}{c}2.1 \\
(0.3-4.0)\end{array}$ \\
\hline
\end{tabular}

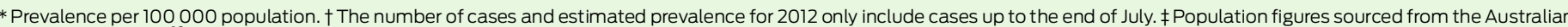
Bureau of Statistics. ${ }^{10}$ 
have comorbidities. Current remote practitioner guidelines recommend performing faecal microscopy for children with failure to thrive, anaemia or chronic diarrhoea, and for adults who are immunosuppressed. ${ }^{8}$ However, there are several potential barriers to testing patients in remote communities, including patient acceptability and the geographical logistics of ensuring a quality specimen reaches the testing laboratory.

Some cases of HWI may have been missed, as data were not available from two providers of pathology services to the NT. Nevertheless, for notifiable diseases in the NT such as hepatitis B, the government and Western Diagnostic Pathology laboratories data capture over $90 \%$ of diagnoses. ${ }^{16}$ We believe it is a reasonable assumption that this will also be the case for HWI. Patients residing in remote areas were well represented in the study population, and annual numbers of faecal microscopy testing were relatively constant over the 10 years. Thus, we are confident that the consistent downward trend in prevalence demonstrated by our data reflects a reduction in the true rate. Finally, although we have shown that most patients with HWI in the NT had anaemia and/or eosinophilia, community controls were not available for comparison to support a causative association.
Our study, despite its limitations, shows that the prevalence of HWI in the NT decreased over a 10-year study period. Infection was rare in children, the target group for the longstanding CCDP, but infection persists in Indigenous adults in remote communities and those homeless in urban centres, and in prisoners. This reflects, at least in part, ongoing health and socioeconomic inequalities in the Indigenous population compared with non-Indigenous Australians. Our study supports the need for continuation of the CCDP, together with improvements in housing, health hardware (such as water supply and waste removal systems) and health promotion. It also emphasises the importance of using albendazole in children and adults with anaemia and/or eosinophilia, with the aim of eventual eradication of human HWI from the NT.

\section{Acknowledgements: We thank Linda Ward and Matthew} Stevens (Menzies School of Health Research) for expert statistical advice.

Competing interests: No relevant disclosures.

Received 2 Nov 2012, accepted 18 Feb 2013.

1 World Health Organization. Soil-transmitted helminthiases: eliminating soil-transmitted helminthiases as a public health problem in children. Progress report 2001-2010 and strategic plan 2011-2020. Geneva: WHO, 2012.

2 Prociv P, Luke RA. The changing epidemiology of human hookworm infection in Australia. Med J Aust 1995; 162:150-154.

3 Brooker S, Bethony J, Hotez PJ. Human hookworm infection in the 21st century. $A d v$ Parasitol 2004; 58: 197-288.

4 Harhay MO, Horton J, Olliaro PL. Epidemiology and control of human gastrointestinal parasites in children. Expert Rev Anti Infect Ther 2010; 8 : 219-234.

5 Taylor-Robinson DC, Maayan N, Soares-Weiser K, et al. Deworming drugs for soil-transmitted intestinal worms in children: effects on nutritional indicators, haemoglobin and school performance. Cochrane Database Syst Rev 2012 (7): CD000371.

6 Currie B. Why does Australia have no national drug policy [letter]? Med J Aust 1992; 157: 210.

7 Victorian Medical Postgraduate Foundation. Antibiotic guidelines. 8th ed. Melbourne: Interprin, 1994.

8 Central Australian Rural Practitioners Association. CARPA standard treatment manual. 5th ed. Alice Springs: CARPA, 2009.

9 Holt DC, McCarthy JS, Carapetis JR. Parasitic diseases of remote Indigenous communities in Australia. Int J Parasitol 2010; 40: 1119-1126.

10 Australian Bureau of Statistics. Australian demographic statistics, Jun 2012. Canberra: ABS, 2012. (ABS Cat. No. 3101.0.) http://www.abs.gov. au/Ausstats/abs@.nsf/mf/3101.0 (accessed Jan 2013).

11 Garcia LS, Bruckner D. Diagnostic medical parasitology. 3rd ed. Washington, DC: ASM Press, 1997: 615-618.

12 Australian Bureau of Statistics. Australian Standard Geographical Classification (ASGC), July 2011. Canberra: ABS, 2011. (ABS Cat. No. 1216.0.) http://www.abs.gov.au/ausstats/ abs@.nsf/Latestproducts/E4A3564F8B855E26 CA25791F000EEC45?opendocument (accessed Jan 2013).

13 Hotez PJ, Brooker S, Bethony JM, et al. Hookworm infection. N Engl J Med 2004; 351: 799-807.

14 Hopkins RM, Gracey MS, Hobbs RP, et al. The prevalence of hookworm infection, iron deficiency and anaemia in an Aboriginal community in north-west Australia. Med J Aust 1997; 166: 241-244.

15 Thompson RCA, Reynoldson JA, Garrow SC, et al. Towards the eradication of hookworm in an isolated Australian community. Lancet 2001; 357: 770-771.

16 Aratchige PE, Markey P, Webby R, Krause V. Hepatitis B in the Northern Territory - an analysis of hepatitis B notifications. NT Dis Control Bull 2012; 19 (2): 1-12. 